\title{
BMJ Open Study protocol for a pilot, randomised, double-blinded, placebo controlled trial of perineural local anaesthetics and steroids for chronic post-traumatic neuropathic pain in the ankle and foot: the PREPLANS study
}

\author{
Anuj Bhatia, ${ }^{1,2}$ Vera Bril, ${ }^{3}$ Richard T Brull, ${ }^{4}$ Anthony Perruccio, ${ }^{5,6}$ \\ Duminda Wijeysundera, ${ }^{1,7}$ Sabbeh Alvi, ${ }^{2}$ Johnny Lau, ${ }^{8}$ Rajiv Gandhi, ${ }^{9}$ \\ Nizar Mahomed, ${ }^{5,10}$ Aileen M Davis ${ }^{5,11}$
}

To cite: Bhatia A, Bril V, Brull RT, et al. Study protocol for a pilot, randomised, double-blinded, placebo controlled trial of perineural local anaesthetics and steroids for chronic posttraumatic neuropathic pain in the ankle and foot: the PREPLANS study. BMJ Open 2016;6:e012293.

doi:10.1136/bmjopen-2016012293

- Prepublication history for this paper is available online. To view these files please visit the journal online (http://dx.doi.org/10.1136/ bmjopen-2016-012293).

Received 13 April 2016 Accepted 25 May 2016

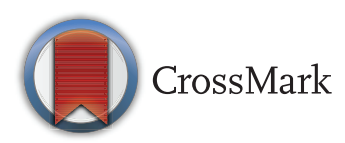

For numbered affiliations see end of article.

Correspondence to Dr Anuj Bhatia; Anuj.Bhatia@uhn.ca

\section{ABSTRACT}

Introduction: Peripheral neuropathic pain (PNP) associated with trauma is often refractory to treatment. Administration of local anaesthetics (LA) and steroids around injured nerves has been proposed as an option for patients unresponsive to conventional treatments for refractory PNP following trauma. There is insufficient evidence to support a large, potentially expensive, full-scale randomised controlled trial (RCT) that involves comparison of effects of perineural steroids and LA against LA or saline injections on analgesia, physical and psychological functioning, and quality of life. There is also a lack of data that would allow estimation of analgesic efficacy or sample size for the full-scale RCT. The objective of this pilot RCT is to yield information to support planning of a full-scale RCT in this population.

Methods and Analysis: 30 participants with posttraumatic PNP in the ankle and foot of moderate-tosevere intensity and duration of more than 3 months will be enrolled in this pilot RCT. Participants will be randomised to receive three ultrasound-guided perineural injections of $0.9 \%$ saline, $0.25 \%$ bupivacaine (a long-acting LA) or a combination of $0.25 \%$ bupivacaine and a steroid (methylprednisolone $16 \mathrm{mg}$ per nerve) at weekly intervals. The primary objectives are to determine the feasibility and sample size of a full-scale RCT in this population. The secondary objectives are to evaluate the effect of study interventions on analgesia, persistence of neuropathic pain, psychological and physical function, quality of life and participants' global impression of change at 1 and 3 months after the interventions. In addition, adverse effects associated with perineural injections and with systemic absorption of steroids will also be recorded.

Ethics and Dissemination: The protocol was approved by the University Health Network Research Ethics Board (UHN REB number 15-9584-A).

\section{Strengths and limitations of this study}

- This is the first study to assess the feasibility of perineural administration of local anaesthetics and steroids with assessment of clinically relevant domains of pain at multiple time points in patients with trauma-related chronic neuropathic pain.

- Information from this study will support planning of a methodologically sound trial that has adequate power to compare analgesic efficacy of perineural steroids against perineural local anaesthetics and steroids in this population, while ensuring standardisation in terms of medication doses and injection techniques.

- Adverse effects following perineural interventions will be systematically evaluated.

- This is a single-centre clinical trial. Challenges with enrolment of patients may require inclusion of other centres to achieve enrolment goals.

- Loss of participants at follow-up is possible, especially for non-responders. Trial enrolment and duration may have to be extended to ensure availability of data for analysis.

The results will be disseminated in peer-reviewed journals and at scientific conferences.

Trial registration number: NCT02680548;

Pre-results.

\section{INTRODUCTION}

The prevalence of chronic pain in the population ranges from $20 \%$ to $35 \%^{1}$ and one in five patients with chronic pain has neuropathic characteristics. This suggests that the population prevalence of neuropathic pain (NP) is about $5-8 \%^{2}$ but some studies have 
reported higher estimates $(17.9 \%){ }^{3} \mathrm{NP}$ can be central or peripheral or a combination of both. Peripheral NP (PNP) is defined as pain caused by a lesion or disease affecting the peripheral somatosensory nervous system. ${ }^{45}$ PNP is present in more than $50 \%$ of patients with NP, with prevalence reported in the range of 2.5$4 \%$ in the general population. ${ }^{2}$ PNP is often severely debilitating; it is largely resistant to treatment, ${ }^{67}$ and its management and sequelae are a significant burden on healthcare resources and society. 89

PNP has multiple aetiologies but trauma is an important cause in the working-age population. ${ }^{10}$ Trauma to the hand and foot (crush injuries, fractures, ligament tears or sprains, surgery) is common in work-related injuries ${ }^{11}$ and can result in chronic pain (ie, pain persisting for more than 3 months) of moderate-to-severe intensity that may be predominantly neuropathic or with mixed neuropathic and nociceptive characteristics. Nerve fibres in lower limbs are often involved in traumatic injuries. The sensory fibres of these nerves display enhanced or aberrant neuronal activity from injured primary afferents and this mechanism contributes to the development of PNP. ${ }^{12}$

\section{Treatment options for PNP}

Current treatment strategies for post-traumatic PNP include pharmacological options, physical therapy and cognitive behavioural interventions. However, a significant number of patients have inadequate pain relief (only one participant has analgesic benefit for every three who receive these treatments) and/or experience adverse effects (cognitive impairment, gastrointestinal problems) from existing oral pharmacological treatments. ${ }^{7}$ Lack of effective therapies necessitate exploration of new strategies to treat chronic PNP. Injections of local anaesthetics (LA) and/or steroids around affected nerves (ie, perineurally), neuromodulation and intrathecal therapies are interventional treatments recommended for managing refractory PNP, but perineural injections are the most cost-effective of these options while being associated with the lowest incidence and severity of adverse effects. ${ }^{13}$

\section{Role of perineural steroids in treatment of peripheral NP}

Nerve injury has been shown to activate secretion of inflammatory mediators and increase ectopic discharge from the injured nerve, ${ }^{6}$ thereby contributing to the development of NP. ${ }^{14}{ }^{15}$ Corticosteroids, through their anti-inflammatory and membrane-stabilising actions, have the potential to reduce post-traumatic PNP. However, systemic administration of steroids is associated with significant adverse effects (AE) and this has led to exploration of the perineural route to maximise therapeutic efficacy while minimising adverse effects. Application of steroids to injured nerve fibres suppresses inflammation and ectopic discharge ${ }^{16}$ thereby alleviating oedema and providing analgesia. Local/perineural delivery of long-acting steroids (duration of action is around 3 months) for treatment of post-traumatic/compression PNP has been studied with inconclusive results. However, in many studies, the nature of pain syndromes was unclear, the injections may not have been accurate because of the lack of image-guidance and the dose of steroids was not clearly specified. ${ }^{17} 18$ Perineural injections of steroids have been used to treat pain from Morton's neuroma (a PNP condition in the foot that is non-traumatic in origin) for 3 months in a randomised controlled trial $^{19}$ and for 9 months in a case series ${ }^{20}$ with significant analgesic benefit in $30-40 \%$ of patients. A combination of steroids and LA agents was found to be more effective than LA alone in decreasing pain intensity, neuropathic symptoms and signs, and analgesic requirements in a randomised controlled trial (RCT) that enrolled patients with post-traumatic PNP following injuries to various peripheral nerves. ${ }^{21}$ However, this trial included patients with a wide spectrum of pain syndromes, factors relevant to pain outcomes (eg, physical and psychological function) were not reported and image guidance was not used during injections. Ultrasound-guided injections of LA around the nerves at the ankle have been used successfully for providing analgesia for operative interventions, ${ }^{22}$ but there is no published literature on the role of ultrasound-guided delivery of perineural LA or steroids (or a combination) in the management of post-traumatic PNP in the ankle and foot. Finally, it also appears that perineural steroids are more effective in subsets of patients when this intervention is provided within 3 years of onset of symptoms, possibly because levels of anxiety or depression are lower and opioid doses are low in the earlier period. ${ }^{23}$

\section{Importance of assessing outcome domains of PNP}

The Initiative on Methods, Measurement, and Pain Assessment in Clinical Trials (IMMPACT) recommendations for evaluation of outcomes in trials of interventions for relieving pain include assessment of intensity of pain, physical and psychological functioning, participant ratings of global improvement and satisfaction with treatment, symptoms and adverse effects. ${ }^{24}$ However, very few trials that evaluated interventions for relieving chronic PNP have included all the relevant outcome domains in their methodology. Further, administration of steroids can result in potentially harmful sequelae that include hyperglycaemia, hypertension, osteoporosis, myopathy, increased susceptibility to infections, psychosis, cataracts and necrosis of the skin; none of the existing trials evaluated these adverse effects as an outcome.

\section{Rationale for this pilot trial}

Knowledge gaps exist for healthcare providers caring for patients with chronic post-traumatic PNP. High-quality evidence is required to compare analgesic efficacy of perineural steroids compared to perineural LA and steroids in this population, while ensuring standardisation in terms of medication doses and injection techniques. Adverse effects following the interventions also need to 
be systematically evaluated. Finally, subsets of patients who are more likely to respond to this approach need to be identified. We have addressed some of these gaps through our previous research efforts-a systematic review and meta-analysis ${ }^{25}$ - and a retrospective observational study of efficacy of perineural steroids and LA in peripheral compression-related or trauma-related neuropathic pain. ${ }^{26}$ An adequately powered RCT that compares placebo or saline injections against LA or steroid injections is appropriate to address the issue of efficacy of perineural steroids for post-traumatic NP, but there is insufficient evidence to support a large, potentially expensive, full-scale RCT at present; nor are there data that would allow estimation of analgesic efficacy or sample size. Therefore, we propose a pilot RCT that will yield information to support planning of a full-scale RCT in this population.

\section{Hypothesis}

There is no hypothesis because this is a pilot RCT to assess the feasibility of a full-scale RCT. ${ }^{27}$

\section{Specific aims and objectives}

This project will be a pilot RCT with the aim of facilitating planning of a full-scale RCT to compare efficacy of image (ultrasound)-guided administration of perineural combination of steroids and LA against perineural LA or $0.9 \%$ sodium chloride (this third arm will help negate the potential placebo analgesic effect of injections) in providing sustained analgesic benefit in participants with chronic NP of the foot and ankle. Primary and secondary objectives of this RCT are listed in box 1 .

\section{METHODS AND ANALYSIS}

\section{Study design}

The SPIRIT (Standard Protocol Items for Randomized Trials) recommendations were followed in preparing the protocol. ${ }^{28} 29$ The study design will be a prospective, randomised, concealed, single centre, parallel group trial with three groups (figure 1) and allocation in equal proportions. Study participants and outcome evaluators will be blinded to treatment assignment.

\section{Enrolment}

All eligible participants with chronic post-traumatic neuropathic pain in the ankle and foot from our outpatient pain clinic who agree to participate in the study and provide informed written consent will be invited to participate in the study. Participants will be recruited via personal correspondence by the research assistant and during sessions in the outpatient department; thus, the initial contact will be in these settings.

\section{Randomisation and concealment of allocation}

Participants will be randomised to the study providing they fulfil the entry criteria at screening (box 2). A computer-generated randomisation sequence will be
Box 1 Primary and secondary objectives. DN4, Doleur Neuropathique 4; NRS, numerical rating score

\section{Primary objectives:}

- To determine the feasibility of a full-scale randomised controlled trial (RCT) based on experience gained from conducting this pilot RCT

- To evaluate the research objectives for a full-scale RCT: information from this pilot RCT may result in modification of hypotheses and outcomes for the full-scale RCT

- To determine the sample size for the full-scale RCT: difference among the three groups in mean numerical rating score (NRS) for pain at 1 month and 3 months after the interventions (effect size) and its variance will help inform the calculation of the sample size for the full-scale RCT with adequate power.

Secondary objectives:

In patients who have trauma or compression-related chronic peripheral neuropathic pain, of moderate-to-severe intensity, in the ankle and/or the foot:

- To evaluate the effect of perineural administration of steroids with local anaesthetics (LA), only LA or $0.9 \%$ sodium chloride on NRS scores for pain (effect size and variance) at one and 3 months after the interventions.

- To evaluate whether perineural administration of steroids with LA, only LA or $0.9 \%$ sodium chloride has an impact on the incidence of neuropathic pain, catastrophising, anxiety, depression, interference with activities, quality of life and lower extremity function at 1 month after the interventions.

- To assess participants' global impression of change at 1 and 3 months after the interventions

- To assess incidence of adverse effects at 1 and 3 months after the interventions

used to assign participants to treatment arms. A block randomisation technique will be used, allowing six participants at a time to be randomised in equal proportions to the three treatment options. The allocation for every participant will be kept in a sealed envelope that will be opened immediately before the intervention by the physician performing the procedure but who is not involved in assessing outcomes of the study. Participants will not be allowed to cross over from one group to another until the end of the study, but they can chose to leave the study at any time if they want to receive the current standard of care (local anaesthetic and steroid injections).

We will assess adequacy of the randomisation process at the follow-up at 3 months after the intervention by asking study participants which group they believe they were assigned to. The participants and the investigator performing post-procedure assessments will be blinded. The physician performing the procedure will not be blinded, but he/she will not be involved in any postprocedure assessment or data collection.

\section{Study overview}

Participation in the study will involve two clinical visits (one before and another at 1 month after the procedures) and three visits for procedures to be performed on a weekly basis. 
Figure 1 Flow diagram for the pilot RCT. RCT, randomised controlled trial.
Post-traumatic chronic (> 3 months) neuropathic pain in the foot with pain NRS $>3 / 10$ and DN4 score $>3 / 10$

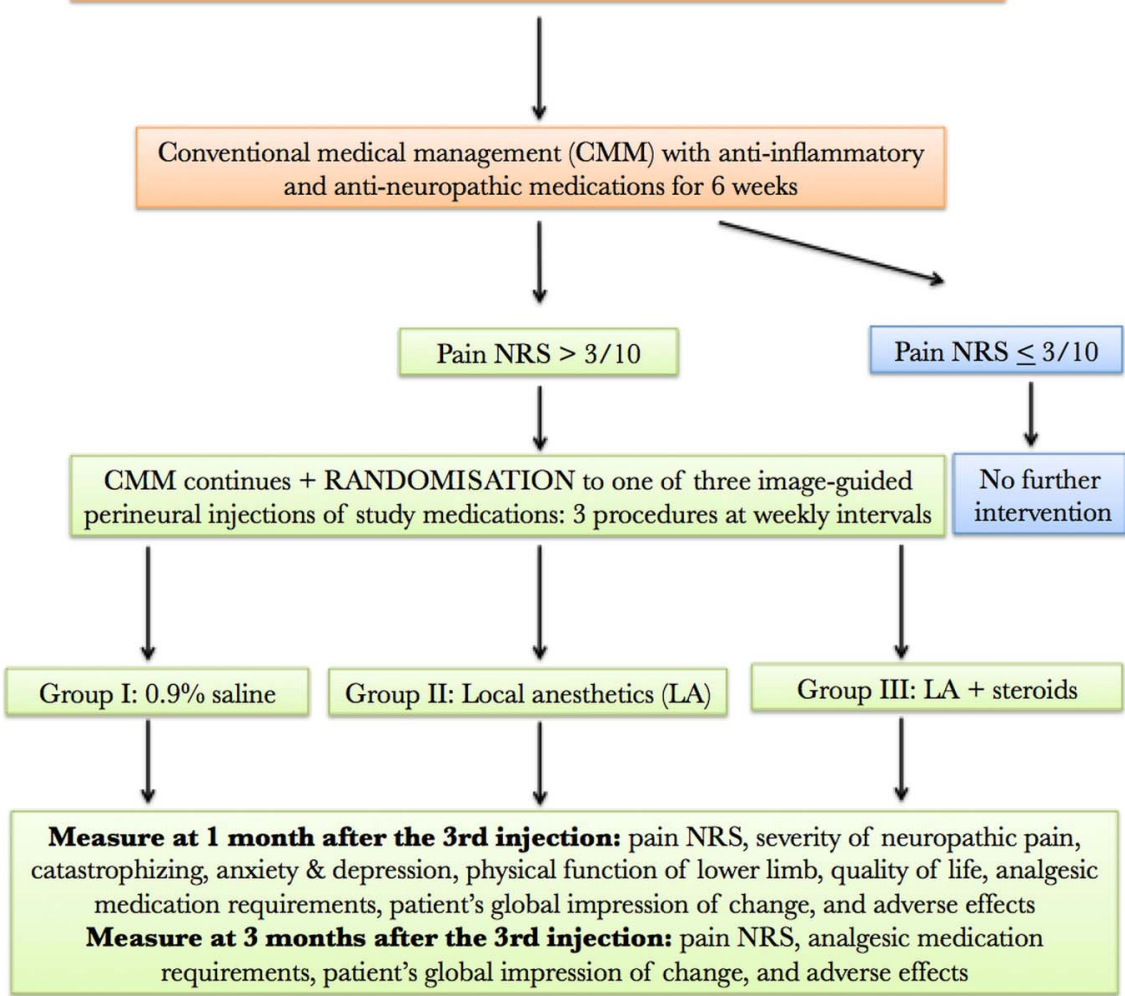

\section{Preprocedure visit}

The baseline visit, within 1 week prior to the first study procedure (perineural injection), will be used to obtain written informed consent, record demographic data (age, gender, height and weight), and measure blood

\section{Box 2 Inclusion and exclusion criteria.}

\section{Inclusion criteria:}

- Pain in foot in a neuroanatomically congruent location following trauma (including surgery) for more than 3 months

- Physician-reported Doleur Neuropathique 4 scoring confirming neuropathic pain (score $\geq 4 / 10$ )

- Average intensity of pain $\geq 4 / 10$ on numerical rating score

- Failed trial of appropriate doses of first-line medications for neuropathic pain (anticonvulsants and/or antidepressants) for 6 weeks

Exclusion Criteria:

- Age 18 years or more

- Perineural or intra-articular steroid injections in the past 6 months

- Allergy to local anaesthetics and/or steroids

- Ongoing litigation issues related to the patient's pain

- Pregnancy

- Coagulopathy or systemic infection

- Peripheral neuropathy or myopathy, central neuropathic pain (eg, post-stroke pain)

- Infection in the ankle or foot

- An unstable medical or psychiatric condition

- Significant catastrophising as indicated by the pain catastrophizing scale score equal to or more than $30 / 52$ pressure and capillary glucose. Duration of pain in months, documentation of name and number of injured nerves, and administration of questionnaires to assess outcomes will also be performed at this visit. Data will be collected for nature of injury (open fracture, closed fracture, soft tissue trauma) and participants' employment status (employed or not). Average intensity of pain in the foot and the ankle will be measured using the numerical rating score (NRS) for pain. The questionnaires will include screening for neuropathic pain with the Doleur Neuropathique 4 (DN4) ${ }^{30}$ and assessment of its severity by the patient-reported Neuropathic Pain Symptom Inventory (NPSI). Psychological function will be assessed through use of the Pain Catastrophizing Score (PCS), ${ }^{31}$ anxiety component of the Hospital Anxiety and Depression Scale (HADS-A), and Patient Health Questionnaire-9 (PHQ-9) for depression; all three are patient-reported outcomes. Physical function will be assessed using the Brief Pain Inventory subscale for Interference with activities (BPI-I), and Lower Extremity Function Score (LEFS), both of which are patient-reported, as is the Short Form-12 (SF-12) questionnaire, a measure of quality of health. Data will also be collected on daily opioid intake measured in oral morphine equivalents (OME), daily doses of gabapentin and amitriptyline or nortriptyline. Cross-sectional area (CSA) and circumference of the tibial nerve will be measured using ultrasound in patients at $3 \mathrm{~cm}$ proximal to the medial malleolus ${ }^{32}$ in whom this nerve is involved in generating neuropathic pain. Change in CSA and 
circumference of the tibial nerve (if any) (as measured by the differences between baseline values and measurements at 1 month following the third procedure) will be correlated with analgesic response to perineural injections because a decrease in these parameters may indicate a reduction in oedema of the nerve.

\section{Procedure visits 1, 2 and 3}

Periprocedure management will be identical to that received by all patients having a perineural injection in the ankle or foot at our clinic. This includes obtaining peripheral intravenous access and application of routine electrocardiogram, non-invasive blood pressure and pulse oximeter monitors. Intravenous midazolam or propofol will be given intravenously for anxiolysis and sedation.

Participants will be randomly assigned to one of the following three groups to receive US-guided injections of the study injectates around one or more of the five nerves innervating the foot and ankle. The study injectates will be $0.9 \%$ saline, or local anaesthetic $(0.25 \%$ bupivacaine, or local anaesthetic $(0.25 \%$ bupivacaine $)$ with steroids (methylprednisolone (Depo-Medrol]) $4 \mathrm{mg} / \mathrm{mLs}$ ). Two to $6 \mathrm{mLs}$ of the study injectate will be injected under ultrasound guidance to surround each of the affected nerves (maximum total injected volume will be $20 \mathrm{mLs}$ ). Nerves to be targeted by the injections will be decided by the treating physician on the basis of the area of the foot and ankle that displays features of neuropathic pain. Each injection of study medications will be preceded by subcutaneous injections of $0.5 \mathrm{~mL}$ of the local anaesthetic (2\% lidocaine) at each of the injection sites. This will reduce the discomfort of the study participants from the injections. In addition, the subcutaneous local anaesthetic will cause numbness of the skin and this will ensure blinding of the participants and outcome assessors regarding group allocation. Three ultrasound-guided procedures will be performed at weekly intervals over 3 weeks. The US-guided technique for performing these procedures has been described in previous studies from our centre. ${ }^{22}$

Following completion of the procedure, participants will be taken to the post-procedure recovery unit. Any complications related to the procedure will be recorded and managed if required. Participants will be discharged from the unit as per routine clinic policy. They will be advised to take 1 or 2 tablets of Percocet (containing $5 \mathrm{mg}$ of oxycodone and $325 \mathrm{mg}$ of acetaminophen) or Tylenol number 3 (containing $30 \mathrm{mg}$ of codeine and $325 \mathrm{mg}$ of acetaminophen) every 6 hours to a maximum of 8 tablets in 24 hours if they have pain scores equal to or higher than 4 of 10. Frequency of use of these medications by study participants will be recorded in a pain diary provided to them and the average daily opioid consumption for the 1 week preceding the follow-ups at 1 and 3 months will be recorded.

\section{Post-procedure visit at 1 month after the third procedure}

This visit will be scheduled at 1 month following the third procedure. Data collection will include blood pressure and capillary glucose values. We will enquire about participants' employment status (employed or not). NRS for pain, DN4, NPSI, PCS, HADS-A, PHQ-9, BPI-I, LEFS and SF-12 will be administered on this visit. Data will also be collected on daily opioid intake measured in OME, daily doses of gabapentin and amitriptyline or nortriptyline. CSA and circumference of the tibial nerve will be measured as described above in participants in whom this nerve is involved in generating NP. Participant's global impression of change (PGIC) for pain on a five-point Likert scale ('significantly worse', 'somewhat worse', 'no change', 'somewhat better' and 'significantly better') will also be assessed. Any evidence of adverse effects including skin necrosis at the site of injections, symptoms of myopathy, psychosis and occurrence of local or systemic infections will be recorded.

\section{Post-procedure visit at 3 months after the third procedure}

This follow-up will be carried out over the telephone. The questionnaires will include NRS for pain, DN4, NPSI and PGIC. Data will also be collected on daily opioid intake measured in OME, daily doses of gabapentin and amitriptyline or nortriptyline. Any evidence of adverse effects will also be recorded.

Information regarding study procedures, interventions and assessments is provided in table 1 .

\section{Concomitant therapies}

Conservative measures (oral analgesics, physiotherapy) will continue during the study. Utilisation of these modalities (medication names and average daily doses over the preceding week, number of physiotherapy visits per week) will be recorded at the baseline and follow-up visits at 1 and 3 months.

\section{Participant withdrawal}

Participants will be asked to contact the investigator if they experience impairment of glycaemic control (in patients with diabetes mellitus), skin necrosis at the site of injections, symptoms of myopathy, psychosis and occurrence of local or systemic infections. On the basis of clinical judgement, the participant may be withdrawn from the study. Participants can also withdraw from the trial at any time to receive the current standard of care (perineural local anaesthetics and steroids). Follow-up consultations and any necessary tests or investigations will be arranged as judged by the investigator.

\section{Discontinuation criteria}

Every effort will be made to retain participants in the trial and to minimise withdrawals. Participants may choose at any time to withdraw from the study. Participants may also be withdrawn from the study after the consent and randomisation process if the procedure cannot be performed for any reason, for example, 
Table 1 Schedule of procedures, interventions and assessments

\begin{tabular}{|c|c|c|c|c|}
\hline Instruments and batteries & Preintervention & $\begin{array}{l}\text { At the time of study } \\
\text { interventions } \\
\text { (1st procedure) }\end{array}$ & $\begin{array}{l}\text { At } 1 \text { month post } \\
\text { intervention } \\
\text { (in the clinic) }\end{array}$ & $\begin{array}{l}\text { At } 3 \text { months post } \\
\text { intervention } \\
\text { (over the phone) }\end{array}$ \\
\hline NRS-ankle/foot pain* & $\mathrm{x}$ & & $\mathrm{x}$ & $\mathrm{x}$ \\
\hline Injured nerves (name and number) ${ }^{\star}$ & $x$ & & & \\
\hline DN4* & $\mathrm{x}$ & & $\mathrm{x}$ & $\mathrm{x}$ \\
\hline NPSI & $x$ & & $\mathrm{x}$ & $x$ \\
\hline PCS $^{*}$ & $\mathrm{x}$ & & $\mathrm{x}$ & \\
\hline SF-12* & $x$ & & $\mathrm{x}$ & \\
\hline BPI-I* & $\mathrm{x}$ & & $\mathrm{x}$ & \\
\hline LEFS $^{*}$ & $\mathrm{X}$ & & $\mathrm{x}$ & \\
\hline HADS & $\mathrm{x}$ & & $\mathrm{x}$ & \\
\hline PHQ-9* & $x$ & & $\mathrm{x}$ & \\
\hline Work status & $\mathrm{x}$ & & $\mathrm{x}$ & $x$ \\
\hline Fasting blood glucose & $\mathrm{X}$ & & $\mathrm{x}$ & \\
\hline CSA of tibial nerve & & $\mathrm{x}$ & & \\
\hline Blood pressure* & $\mathrm{x}$ & & $\mathrm{x}$ & \\
\hline PGIC & & & $x$ & $x$ \\
\hline Adverse effects $\dagger$ & & & $\mathrm{X}$ & $\mathrm{x}$ \\
\hline Age $^{\star}$ & $x$ & & & \\
\hline Height and weight* & $\mathrm{x}$ & & & \\
\hline Gender* & $x$ & & & \\
\hline Nature of injury (no /closed/open fracture) ${ }^{\star}$ & $\mathrm{x}$ & & & \\
\hline Duration of pain (months) $)^{*}$ & $x$ & & & \\
\hline Daily OME $(\mathrm{mg})^{*}$ & $\mathrm{x}$ & & $\mathrm{x}$ & $\mathrm{x}$ \\
\hline Daily gabapentin dose $(\mathrm{mg})^{\star}$ & $x$ & & $x$ & $\mathrm{x}$ \\
\hline Daily amitriptyline dose $(\mathrm{mg})^{\star}$ & $\mathrm{x}$ & & $x$ & $x$ \\
\hline
\end{tabular}

abnormal anatomy precluding safe performance of the procedure. Apparent block failure is not an indication for withdrawal. Reasons for withdrawal will be documented. Data accumulated up to the time of withdrawal will be retained and included in subsequent analyses. Data will be analysed on an intention-to-treat basis.

\section{Safety considerations}

Adverse effects of perineural injections include increased pain at the site of injection, bruising and injury to the nerves resulting in temporary or permanent sensory and/or motor loss. Steroids injected around nerves can be absorbed systemically and cause hypertension, hyperglycaemia, myopathy, osteoporosis, psychosis, cataracts and reduced immunity. In the present study, the doses of injected steroids are low and severe adverse effects are unlikely to be seen. Adverse effects will be assessed on the basis of changes in measured values and qualified as significant based on predefined cut-off values (elevation of systolic blood pressure by $30 \%$ or more compared to baseline and elevation of capillary blood glucose measurements by $50 \%$ or more at 1 month after the third procedure). Other adverse effects related to perineural injections or those related to systemic absorption of steroids will be qualified as present or absent at 1 and 3 months after the third procedure.

\section{Outcomes}

IMMPACT $^{24}$ and CONSORT ${ }^{33}$ guidelines for data collection will be followed. The primary outcome of this pilot RCT will be parameters that determine feasibility of a full-fledged RCT with the same interventions, inclusion and exclusion criteria. Feasibility will be assessed through assessment of the multiple variables. The secondary outcomes include measurement of scores for intensity and neuropathic character of pain, anxiety, depression, catastrophising, quality of life, physical functioning and analgesic requirements. Details are provided in box 3 .

\section{Sample size}

Thirty participants will be enrolled in this study with 10 participants in each group. To account for an estimated $10 \%$ loss to follow-up, we will enrol 33 participants. Around 12 patients undergo this intervention in a month at our pain clinic. Assuming an enrolment rate of $75 \%$ and a follow-up period of 3 months, this pilot 


\section{Box 3 Primary and secondary outcomes.}

\section{Primary Outcomes}

- Enrolment and retention of participants: proportion of potential participants who consent to be randomised and proportion of randomised participants who drop out or are lost to follow-up will help in determining the number of participants who need to be enrolled and the duration required to ensure that the desired sample size is achieved for the full-scale randomised controlled trial (RCT). We will aim for the following goals:

- Enrolment of at least $75 \%$ of participants who are approached with a proposal to participate

- Enrolment of at least nine participants per month

- Retention until the end of the study of at least $90 \%$ of participants who are enrolled

Success at achieving all three goals will signify that the full-scale RCT is 'definitely feasible'; achieving two of the three goals will indicate that it is 'probably feasible', and achieving one or none of the goals will signify that the full-scale RCT is 'not feasible'.

- Randomisation techniques and its attendant challenges

- Appropriateness of eligibility (inclusion and exclusion) criteria

- Assessment procedures for capturing data: development of case record forms, training of research staff in data collection, appraisal of participant assessment burden

- Acceptability and implementation of the interventions

- Evaluation of costs: expenditure of the pilot RCT will help in planning the budget for the full-scale RCT

Secondary Outcomes

- To determine measures of central tendency and spread (mean and SD) of the numerical rating score (NRS) (range 0-10) for the foot and ankle at 1 and 3 months after the intervention.

- To measure change in the Pain Catastrophizing Score (PCS), Dolores Neuroapthique (DN4), Neuropathic Pain Symptom Inventory (NPSI), anxiety component scores on the Hospital Anxiety and Depression Scale (HADS) and depression scores on the Patient Health Questionnaire-9 (PHQ-9) at 1 month after the intervention compared to preintervention scores.

- To measure change in Brief Pain Inventory interference with activities (BPI-I), Short Form-12 (SF-12) and Lower Extremity Function Score (LEFS) at 1 month after the intervention compared to preintervention scores.

- To evaluate the impact of study interventions on requirement of opioids (measured as average daily oral morphine equivalents in $\mathrm{mg}$ ) and neuropathic medications (average daily doses of gabapentin and/or amitriptyline in $\mathrm{mg}$ )

- To measure incidence of the following adverse effects:

- Hyperglycaemia (change in blood glucose levels) and hypertension (change in blood pressure levels) at 1 month after the intervention compared to preintervention scores.

- To measure incidence of infections at the injection site, skin discolouration or atrophy at the injection site, fractures and evidence of myopathy at 1 month after the intervention.

RCT will require $\sim 9$ months for conduct and data collection. To increase participants' likelihood of enrolment in the trial, the current standard of care (perineural injection of steroids and LA) will be offered to all participants in the $0.9 \%$ sodium chloride and LA groups after completion of the trial follow-up period.
Enrolment of participants and all study procedures will take place at the pain clinic at Toronto Western Hospital, University Health Network. Our centre is one of a few leading academic centres in the world that has the technical expertise, equipment set-up and clinical volumes needed to carry out the proposed study. Patients are referred to these clinics by family physicians and other specialists (neurologists, orthopaedic surgeons) who are involved in the care of patients with chronic post-traumatic neuropathic pain in the ankle and foot.

\section{Data analyses}

A biostatistician will be consulted to assist in data analysis and interpretation. Descriptive analysis will be performed for all data including means and SD or medians for continuous variables and proportions for categorical variables. Pain outcomes by treatment groups will be presented with a variety of $(75 \%, 85 \%$ and $95 \%)$ CIs to describe the range of effects.

\section{Dissemination}

The results of this study will be reported in international peer-reviewed journals in the field of anaesthesiology and pain, and presented at conferences. Participants will be informed of the results of the trial by the investigators. Authorship will be ascribed in accordance with the Vancouver system.

\section{Author affiliations}

${ }^{1}$ Department of Anesthesia and Pain Management and Institute of Health Policy, Management and Evaluation, University of Toronto, Toronto, Ontario, Canada

${ }^{2}$ Department of Anesthesia and Pain Management, University Health NetworkToronto Western Hospital, Toronto, Ontario, Canada

${ }^{3}$ Department of Neurology, University Health Network and Mount Sinai Hospital, Toronto, Ontario, Canada

${ }^{4}$ Department of Anesthesia, University Health Network-Toronto Western Hospital and Institute of Medical Science, University of Toronto, Toronto, Ontario, Canada

${ }^{5}$ Health Care and Outcomes Research, Krembil Research Institute, University Health Network, Toronto, Ontario, Canada

${ }^{6}$ Institute of Health Policy, Management \& Evaluation, and Toronto Musculoskeletal Centre, University of Toronto, Toronto, Ontario, Canada ${ }^{7}$ Department of Anesthesia, University Health Network-Toronto General Hospital, University of Toronto, Toronto, Ontario, Canada

${ }^{8}$ Division of Orthopaedic Surgery, Foot and Ankle Program, Toronto Western Hospital, University Health Network, Toronto, Ontario, Canada

${ }^{9}$ Division of Orthopaedic Surgery, Arthritis Program, Toronto Western Hospital, University Health Network, Toronto, Ontario, Canada

${ }^{10}$ Division of Orthopaedic Surgery, Department of Surgery and Institute of Health Policy, Management and Evaluation, University of Toronto, Toronto, Ontario, Canada

${ }^{11}$ Department of Physical Therapy and Surgery, Graduate Department of Rehabilitation Science, the Institute of Health Policy, Management and Evaluation and Institute of Medical Science, Toronto, Ontario, Canada

Contributors AB, VB, RTB, AP, DW, JL, RG, NM and AMD conceived and designed the study and participated in logistical planning of the study. $A B$ and $\mathrm{SB}$ are responsible for data acquisition. All authors made significant contributions to the development and conceptualisation of the protocol. They also reviewed the draft versions of this paper and have read and approved the final manuscript. 
Funding The study is conducted as an investigator-initiated study with internal funding from the Department of Anesthesia and Pain Management at University Health Network-Toronto Western Hospital. University Health Network is the trial sponsor

Competing interests None declared.

Ethics approval The protocol is approved by the University Health Network Research Ethics Board (UHN REB number 15-9584-A and ClinicalTrials.gov identifier: NCT02680548).

Provenance and peer review Not commissioned; externally peer reviewed.

Open Access This is an Open Access article distributed in accordance with the Creative Commons Attribution Non Commercial (CC BY-NC 4.0) license, which permits others to distribute, remix, adapt, build upon this work noncommercially, and license their derivative works on different terms, provided the original work is properly cited and the use is non-commercial. See: http:// creativecommons.org/licenses/by-nc/4.0/

\section{REFERENCES}

1. Reitsma M, Tranmer JE, Buchanan DM, et al. The epidemiology of chronic pain in Canadian men and women between 1994 and 2007 longitudinal results of the National Population Health Survey. Pain Res Manag 2012;17:166-72.

2. Bouhassira $D$, Lantéri-Minet $M$, Attal $N$, et al. Prevalence of chronic pain with neuropathic characteristics in the general population. Pain 2008;136:380-7.

3. Toth $\mathrm{C}$, Lander J, Wiebe S. The prevalence and impact of chronic pain with neuropathic pain symptoms in the general population. Pain Med 2009;10:918-29.

4. Treede RD, Jensen TS, Campbell JN, et al. Neuropathic pain: redefinition and a grading system for clinical and research purposes. Neurology 2008:70:1630-5.

5. Haanpaa M, Treede RD. Diagnosis and classification of neuropathic pain. Pain Clinical Updates 2010;18:1-6.

6. Moalem G, Tracey DJ. Immune and inflammatory mechanisms in neuropathic pain. Brain Res Rev 2006;51:240-64.

7. Harden RN. Chronic neuropathic pain. Mechanisms, diagnosis, and treatment. Neurologist 2005;11:111-22.

8. Attal N, Lanteri-Minet M, Laurent B, et al. The specific disease burden of neuropathic pain: results of a French nationwide survey. Pain 2011;152:2836-43.

9. Turk DC. Clinical effectiveness and cost-effectiveness of treatments for patients with chronic pain. Clin J Pain 2002;18:355-65.

10. Kehlet $\mathrm{H}$, Jensen TS, Woolf CJ. Persistent postsurgical pain: risk factors and prevention. Lancet 2006;367:1618-25.

11. Lander L, Shah RK, Li Y, et al. Healthcare cost usage for hospitalised injuries sustained in industrial settings in the USA Inj Prev 2013;19:112-18.

12. Zimmermann M. Pathobiology of neuropathic pain. Eur $J$ Pharmaco 2001:429:23-37.

13. Van Zundert J, Hartrick C, Patijn J, et al. Evidence-based interventional pain medicine according to clinical diagnoses. Pain Pract 2011:11:423-9.
14. Sommer C, Kress M. Recent findings on how proinflammatory cytokines cause pain: peripheral mechanisms in inflammatory and neuropathic hyperalgesia. Neurosci Lett 2004;361:184-7.

15. Zhang JM, An J. Cytokines, inflammation, and pain. Int Anesthesiol Clin 2007; 45:27-37.

16. Johansson A, Bennett GJ. Effect of local methylprednisolone on pain in a nerve injury model. A pilot study. Reg Anesth 1997;22:59-65.

17. Wang JC, Chiou HJ, Lu JH, et al. Ultrasound-guided perineural steroid injection to treat intractable pain due to sciatic nerve injury. Can J Anaesth 2013;60:902-6.

18. Johansson A, Sjölund B. Nerve blocks with local anesthetics and corticosteroids in chronic pain: a clinical follow-up study. J Pain Symptom Manage 1996;11:181-7.

19. Thomson CE, Beggs I, Martin DJ, et al. Methylprednisolone injections for the treatment of Morton neuroma: a patient-blinded randomized trial. J Bone Joint Surg Am 2013;95:790-8.

20. Markovic M, Crichton $\mathrm{K}$, Read JW, et al. Effectiveness of ultrasound-guided corticosteroid injection in the treatment of Morton's neuroma. Foot Ankle Int 2008;29:483-7.

21. Eker HE, Cok OY, Aribogan A, et al. Management of neuropathic pain with methylprednisolone at the site of nerve injury. Pain Med 2012;13:443-51.

22. Chin KJ, Wong NW, Macfarlane AJ, et al. Ultrasound-guided versus anatomic landmark-guided ankle blocks: a 6-year retrospective review. Reg Anesth Pain Med 2011;36:611-18.

23. Tan HL, Bhatia A, Gordon A, et al. A retrospective review of role of pudendal nerve blocks in management of chronic pelvic and urogenital pain. http://www.painmed.org/2013posters/abstract-214/ (accessed on 30 July 2014).

24. Dworkin $\mathrm{RH}$, Turk DC, Wyrwich $\mathrm{KW}$, et al. Interpreting the clinica importance of treatment outcomes in chronic pain clinical trials: IMMPACT recommendations. J Pain 2008;9:105-21.

25. Bhatia A, Flamer D, Shah PS. Perineural steroids for trauma and compression-related peripheral neuropathic pain: a systematic review and meta-analysis. Can J Anaesth 2015;62:650-62.

26. Bhatia A, Lau J, Davis AM, et al. Evaluation of efficacy of perineural steroids and local anesthetics for chronic post-traumatic neuropathic pain. Presented as a poster at the 5th International Congress on Neuropathic Pain. France: Nice, 2015. http://neupsig.kenes.com/ scientific-information/scientific-programe.

27. Thabane L, Ma J, Chu R, et al. A tutorial on pilot studies: the what, why and how. BMC Med Res Methodol 2010;10:1

28. Chan AW, Tetzlaff JM, Altman DG, et al. SPIRIT 2013 Statement: defining standard protocol items for clinical trials. Ann Intern Med 2013;158:200-7.

29. Chan AW, Tetzlaff JM, Gøtzsche PC, et al. SPIRIT 2013 explanation and elaboration: guidance for protocols of clinical trials. BMJ 2013;346:e7586.

30. Bouhassira D, Attal N, Alchaar H, et al. Comparison of pain syndromes associated with nervous or somatic lesions and development of a new neuropathic pain diagnostic questionnaire (DN4). Pain 2005;114:29-36.

31. Sullivan MJL, Bishop SR, Pivik J. The pain catastrophizing scale: development and validation. Psychol Assess 1995;7:524-32.

32. Riazi S, Bril V, Perkins BA, et al. Can ultrasound of the tibial nerve detect diabetic peripheral neuropathy? A cross-sectional study. Diabetes Care 2012;35:2575-9.

33. Schulz KF, Altman DG, Moher D. Consort 2010 statement: updated guidelines for reporting parallel group randomized trials. Ann Intern Med 2010;152:726-32. 
Correction: Study protocol for a pilot, randomised, double-blinded, placebo controlled trial of perineural local anaesthetics and steroids for chronic posttraumatic neuropathic pain in the ankle and foot: the PREPLANS study

Bhatia A, Bril V, Brull RT, et al. Study protocol for a pilot, randomised, doubleblinded, placebo controlled trial of perineural local anaesthetics and steroids for chronic posttraumatic neuropathic pain in the ankle and foot: the PREPLANS study. BMJ Open 2016;6:e012293. The first name of the sixth author was misspelled; the correct spelling is Sabeeh. In the Contributors section this author's initials should be $\mathrm{SA}($ not SB).

Open Access This is an Open Access article distributed in accordance with the Creative Commons Attribution Non Commercial (CC BY-NC 4.0) license, which permits others to distribute, remix, adapt, build upon this work noncommercially, and license their derivative works on different terms, provided the original work is properly cited and the use is non-commercial. See: http://creativecommons.org/licenses/by-nc/4.0/

BMJ Open 2016;6:e012293corr1. doi:10.1136/bmjopen-2016-012293corr1 